\title{
Human Brain Myelination from Birth to 4.5 Years
}

\author{
B. Aubert-Broche, V. Fonov, I. Leppert, G.B. Pike, and D.L. Collins \\ Montreal Neurological Institute, McGill University, Montreal, Canada
}

\begin{abstract}
The myelination of white matter from birth through the first years of life has been studied qualitatively and it is well know the myelination occurs in a orderly and predictable manner, proceeding in a caudocranial direction, from deep to superficial and from posterior to anterior. Even if the myelination is a continuous process, it is useful to characterize myelination evolution in normal brain development in order to better study demyelinating diseases. The quantification of myelination has only been studied for neonates. The original contribution of this study is to develop a method to characterize and visualize the myelination pattern using MRI data from a group of normal subjects from birth to just over 4 years of age. The method includes brain extraction and tissue classification in addition to the analysis of $\mathrm{T} 2$ relaxation times to attempt to separate myelinated and unmyelinated white matter. The results agree previously published qualitative observations.
\end{abstract}

\section{Introduction}

The myelination of white matter (WM) allows the transmission of neural impulses through the nervous system. At birth, the progress of human brain myelination has just started. During the first year, myelin spreads through the entire brain. From visual inspection of T1 and T2-weighted data from 82 infants ranged in age from 4 days to 2 years, Barkovich et al. [1 qualitatively described the myelination as occurring in an orderly manner, commencing in the brain stem and progressing to the cerebellum and the cerebrum, and generally proceeding from central to peripheral, from inferior to superior and from posterior to anterior.

At birth, the global appearance of T1-weighted images is similar to that of T2-weighted images in adults: WM has a lower signal intensity than the gray matter (GM). During the following months, the intensity of WM increases with the myelination progression. At the end of the first year, the adult contrast is reached almost everywhere in the brain. The opposite pattern is seen on the T2-weighted images where the appearance of the newborn brain is similar to the adult T1-weighted image. Progressive myelination is seen as a decrease of the WM intensity. Figure 1 shows examples of T1 and T2-weighted images with myelinated and non-myelinated WM regions. Contributing factors to these changes in signal intensity may be a decrease in free water and total water content and an increase in cholesterol and glycolipids [12]. 


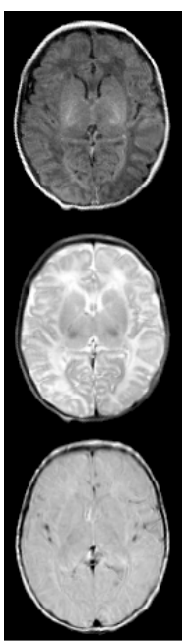

(A)

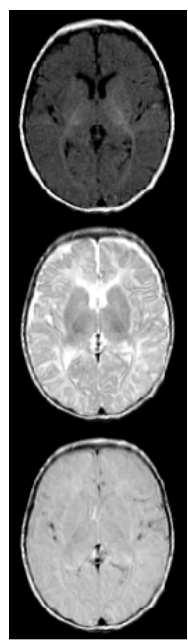

(B)

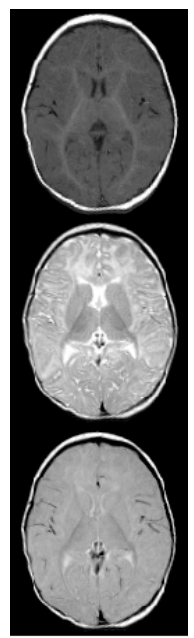

(C)

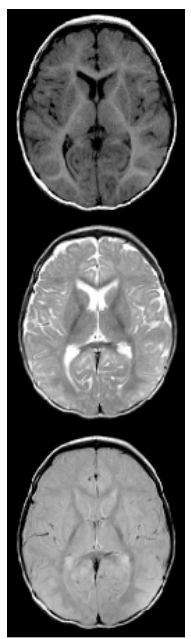

(D)

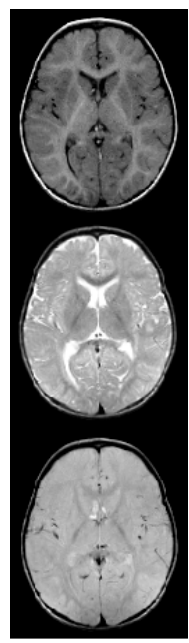

(E)

Fig. 1. Axial T1 (top),T2 (middle) and PD (bottom) weighted from infants at the age of (A) 1 week (B) 3.6 months (C) 6.8 months (D) 9.4 months and (E) 12.3 months

Because of the aforementioned differences in tissue contrast, analysis pipelines designed for use with adult brain are not directly transferable to the unmyelinated or incompletely myelinated brain. The aim of this study is to develop an automated methodology, applicable to pediatric data, which is able to characterize the evolution of the white matter myelination from birth to just over 4 years old and to compare these results with that of Barkovich.

Classifying the various brain tissues (WM, GM and CSF) is of significant interest to provide quantitative information, including the changes in brain volumes during brain development. Tissue classification is a required step in our methodology, and must be applicable to data from any age range, regardless of the stage of myelination. Previous studies have developed techniques for infant brain tissue classification, but they have been applied to MR data from infants only at precise ages (e.g., neonatal infants [3], 4] or 12 and 24 month old infants 5]). Murgasova et al. 5] classified MR images from 1 and 2 year old children using a probabilistic atlas specifically for 1 and 2 year olds. Gildmore et al. 4] use a EM classification with a coregistered probabilistic brain atlas as a prior. To our knowledge, no techniques have been developed to analyse longitudinal data. At a precise time, the classification is easier, as it can be driven by an age-specific prior. We attempt to circumvent this issue by taking advantage of the MR properties of myelinated tissue and to propose subject-specific prior for each subject of the longitudinal study.

When myelination starts, the WM contains only a few thin myelin sheats. Myelination is a progressive process, as it advances the number and the thickness of myelin sheats increase. MR can be used to characterize myelination. Relaxometry is a quantitative imaging technique used to compute MR relaxation 
times for different tissues within the brain. Measurement of relaxation times can give a sensitive and consistent assessment of brain myelination. Because the rate of T2 shortening is much faster than that observed for T1, Barkovich et al. [1] concluded that T2 is more sensitive to tissue changes and is therefore preferred as an index of early brain development. As the reduction of the T2 relaxation time of the WM reflects the progress of myelination, we take advantage of the estimated T2 relaxation value to differentiate between myelinated and unmyelineted WM. The use of relaxometry data to create subject-specific priors for brain tissue classification is the major contribution of this study.

\section{Methods}

Data. The NIH MRI Study of Normal Brain Development represents a unique multi-institutional effort to establish a population-based, representative database of MR imaging and neurocognitive measures in normal infants and children 6. This NIH-funded pediatric database (NIHPD) project provides a publicly available normative pediatric MRI brain and behavioural database, which can subsequently be used in studies assessing normal brain development and deviation due to neurological disorders. From the NIHPD database, we studied 149 acquisitions from an initial sample of 106 normal, healthy children from 0 to 4 years 5 months of age. Each child was scanned between one and six times. The age distribution is shown in Figure 2 .

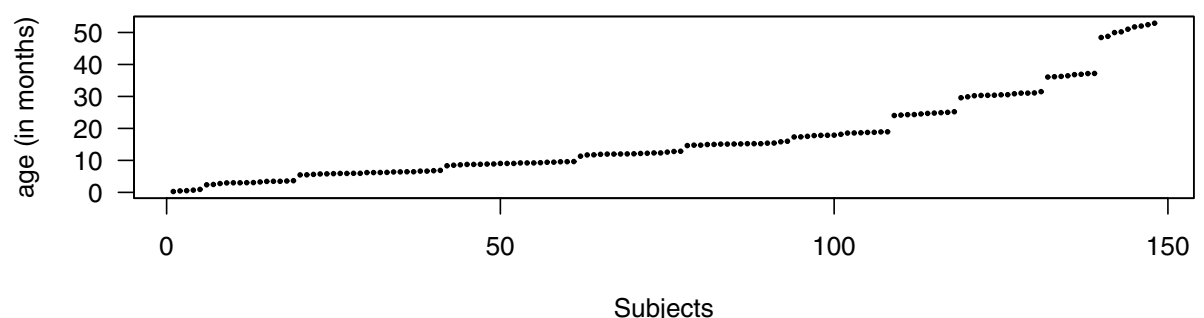

Fig. 2. Age distribution

Data was acquired on $1.5 \mathrm{~T}$ scanners from two different vendors (General Electric and Siemens). All children were scanned during natural sleep (i.e. without sedation). The MR pulse sequences, timing parameters and image resolution are selected so that comparable image contrast and quality is achieved across both scanners. The acquisitions consist of anatomical T1-weighted (2D T1w multislice spin echo $[\mathrm{TR}=500 \mathrm{~ms}, \mathrm{TE}=12 \mathrm{~ms}]$ with a $1^{*} 1^{*} 3 \mathrm{~mm}$ spatial resolution), proton density and T2-weighted (2D T2w Fast/Turbo spin echo with a $1^{*} 1 * 3 \mathrm{~mm}$ spatial resolution $[\mathrm{TR}=3500 \mathrm{~ms}, \mathrm{TE}=14 / 112 \mathrm{~ms}$ for $\mathrm{GE}$ and $13 / 121 \mathrm{~ms}$ for Siemens]), followed by an additional dual echo pair to compute T2 relaxometry (PD/T2w with longer $\mathrm{TE}[\mathrm{TR}=3500 \mathrm{~ms}, \mathrm{TE}=83 / 165 \mathrm{~ms}])$. Not all children remained asleep for the entire protocol, and thus some children do not have the second dual echo 
pair. Figure 1 shows examples of T1,T2 and PD-weighted data of infants from 1 week to 12 months.

Preprocessing and Registration. Intensity non-uniformity in MR images is reduced by applying a $3 \mathrm{D}$ non-uniformity correction algorithm based on the deconvolution of the non-uniformity blurring kernel from the intensity histogram of the image 7. This process, independent of pulse sequence, is applied individually to all native T1, T2 and PD-weighted MR images (Note that this data is used for registration, tissue classification and visualization; it is not used for relaxometry estimation described below). An optimized blockwise non local means denoising filter [8] is also applied to denoise the data.

Eight age-related brain templates are used to provide a registration target $(0-2$ months, 1-5 months, 4-10 months, 8-16 months, 14-22 months, 20-32 months, 2844 months and 40-56 months). For each set of images, T1, T2 and PD-weighted images were non-linearly registered [9] and resampled in the space of the agerelated brain templates, onto a $1 \mathrm{~mm}^{3}$ grid.

Relaxometry. The spin echo signal intensity $S_{i}$ is a function of $S_{i}=S_{0} e^{-\frac{T E_{i}}{T 2}}$ where $S_{0}$ is the equilibrium signal, $S_{i}$ is the signal at the ith echo time $\left(T E_{i}\right)$. T2 maps were estimated on native data using either 2 or 4 echo times:

$$
\ln \left(S_{i}\right)=\ln \left(S_{0}\right)-\frac{T E_{i}}{T 2}
$$

Classification. All T1, T2 and PD-weighted images were averaged to create T1, $\mathrm{T} 2$ and PD-weighted average volumes respectively. These multi-spectral brain images were classified in three fuzzy classes (WM, GM and CSF) with an ANN classifier and a training set from the ICBM adult brain atlas [10, registered to the pediatric targets. For each of the three fuzzy volumes (thresholded at $85 \%$ to have mostly pure tissue), 2000 random voxels were taken to create a new training set. From this training set, a few manual modifications were made to remove some incorrectly classified voxels and to divide the GM into cortical GM and central GM. The result is a pediatric-specific stereotaxic training set that is used in a classification procedure similar to that described by Cocosco [1].

During the subsequent classification of each subject, the standard training data set is modified to customize it for the subject. First, the voxels outside the subject's brain are removed. Second, the WM voxels are divided in two groups depending on the T2 relaxometry map of this subject. For the WM voxels of the training set, if the T2 value is inferior to $160 \mathrm{~ms}$, the voxel is considered as myelinated WM. If the T2 is superior to this value, the voxel is considered as unmyelinated WM. While this threshold is somewhat arbitrary, it is based on the work of Ding et al. [12 and that of Leppert et al. 13] and should enable an approximate separation between myelinated and unmyelinated WM. Even though the actual value may be incorrect, it should permit visualization of the evolving front of myelination, with proper relative timing information between regions and only some (hopefully) small error in absolute timing. 
The resulting training set contains 5 classes (CSF, GM cortical, GM central, WM myelinated, WM unmyelinated) and is used on the T1, T2 and PD-weighted images of the subject to classify these 5 classes with a fuzzy bayesian classifier. Fuzzy classification allows the cleaning of the training set by rejecting voxels if i) the class is different from the assigned class, ii) the fuzzy class value of this voxel is inferior to 0.5 and iii) the difference between the two highest fuzzy classes values is inferior to 0.1 . The "cleaned" training set is used on the T1, T2 and PD-weighted images of the subject to re-classify these 5 classes.

\section{Results}

The reliability of classification depends on correct prior information. For each class and for each subject, the mean and the standard deviation were computed from the training set and the cleaned training set. Table 1 shows the mean of these values for all subjects. We can see that, except for the CSF, the mean is almost the same before and after the cleaning, and the standard deviation

Table 1. With all the voxels of the training set (TS) and the cleaned training set (CTS), a mean and standard deviation were computed for each class and for each subject. This table summarizes the mean for all subjects as well as the mean of the standard deviations.

\begin{tabular}{|c|c|c|c|c|c|c|c|c|c|c|}
\hline & \multicolumn{2}{|c|}{$\mathrm{CSF}$} & \multicolumn{2}{|c|}{$\begin{array}{c}\text { cortical } \\
\text { GM }\end{array}$} & \multicolumn{2}{|c|}{$\begin{array}{c}\text { central } \\
\text { GM }\end{array}$} & \multicolumn{2}{|c|}{$\begin{array}{c}\text { myelinated } \\
\text { WM }\end{array}$} & \multicolumn{2}{|c|}{$\begin{array}{c}\text { unmyelinated } \\
\text { WM }\end{array}$} \\
\hline & TS & CTS & $\mathrm{TS}$ & CTS & TS & CTS & TS & CTS & TS & CTS \\
\hline & & & & & & & & & & \\
\hline & std & $\pm \mathrm{std}$ & \pm std & \pm std & \pm std & $\pm \mathrm{std}$ & $\pm \mathrm{std}$ & $\pm \mathrm{std}$ & \pm std & $\pm \mathrm{std}$ \\
\hline \multirow[t]{2}{*}{$\overline{\mathrm{T} 1 \mathrm{w}}$} & 36.17 & 35.79 & 50.29 & 49.81 & 53.05 & 53.05 & 61.13 & 61.91 & 53.05 & 53.05 \\
\hline & .22 & 8.15 & & 3.1 & 2.22 & 1.9 & 5.16 & 3.73 & 2.2 & 1.91 \\
\hline \multirow[t]{2}{*}{$\mathrm{T} 2 \mathrm{w}$} & 0.73 & 80.74 & 74.07 & 74.61 & 65.84 & 65.43 & 61.95 & 60.93 & 65.84 & 65.43 \\
\hline & 26.08 & 18.92 & 7.46 & 5.53 & 4.41 & 3.61 & 6.13 & 5.52 & 4.41 & 3.61 \\
\hline \multirow[t]{2}{*}{$\overline{\mathrm{PDw}}$} & 59.24 & 65.16 & 75.70 & $\overline{75.74}$ & 77.63 & 77.73 & 71.90 & 71.57 & $\overline{77.63}$ & $\overline{77.73}$ \\
\hline & 17.12 & .58 & 4.28 & 3.00 & 1.65 & 1.44 & 3.33 & 2.80 & 1.656 & 1.44 \\
\hline
\end{tabular}

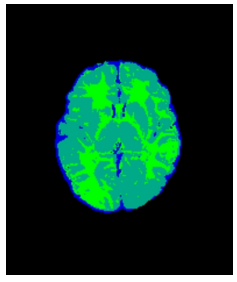

(A)

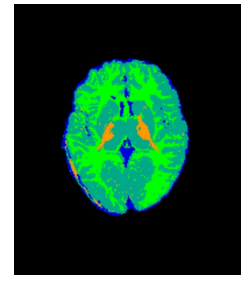

(B)

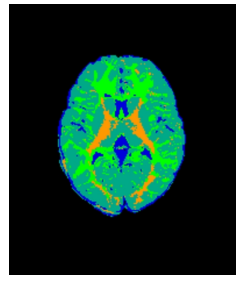

(C)

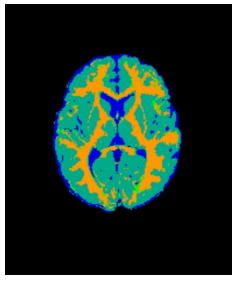

(D)

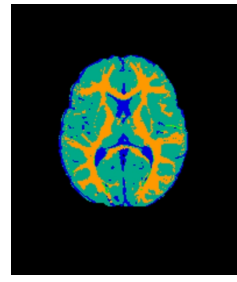

$(\mathrm{E})$

Fig. 3. Axial slices of classified brain volume from (A) 1 week (B) 3.6 months (C) 6.8 months (D) 9.4 months and (E) 12.3 months old infants. Cortical and central GM are represented in blue, unmyelinated WM in green, myelinated WM in orange and CSF in dark blue. 


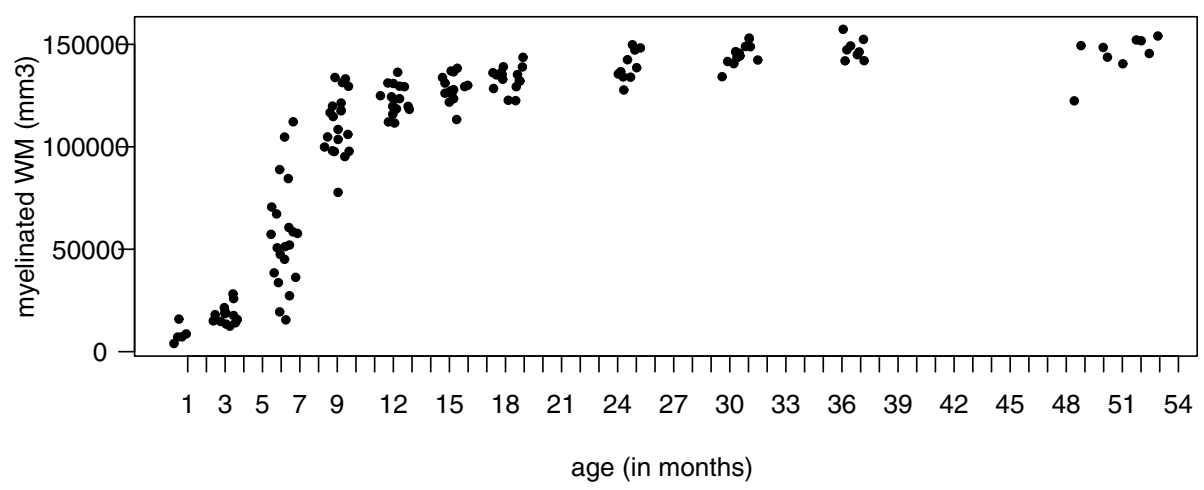

Fig. 4. Evolution of the myelinated WM volume from birth to 4 years 5 months

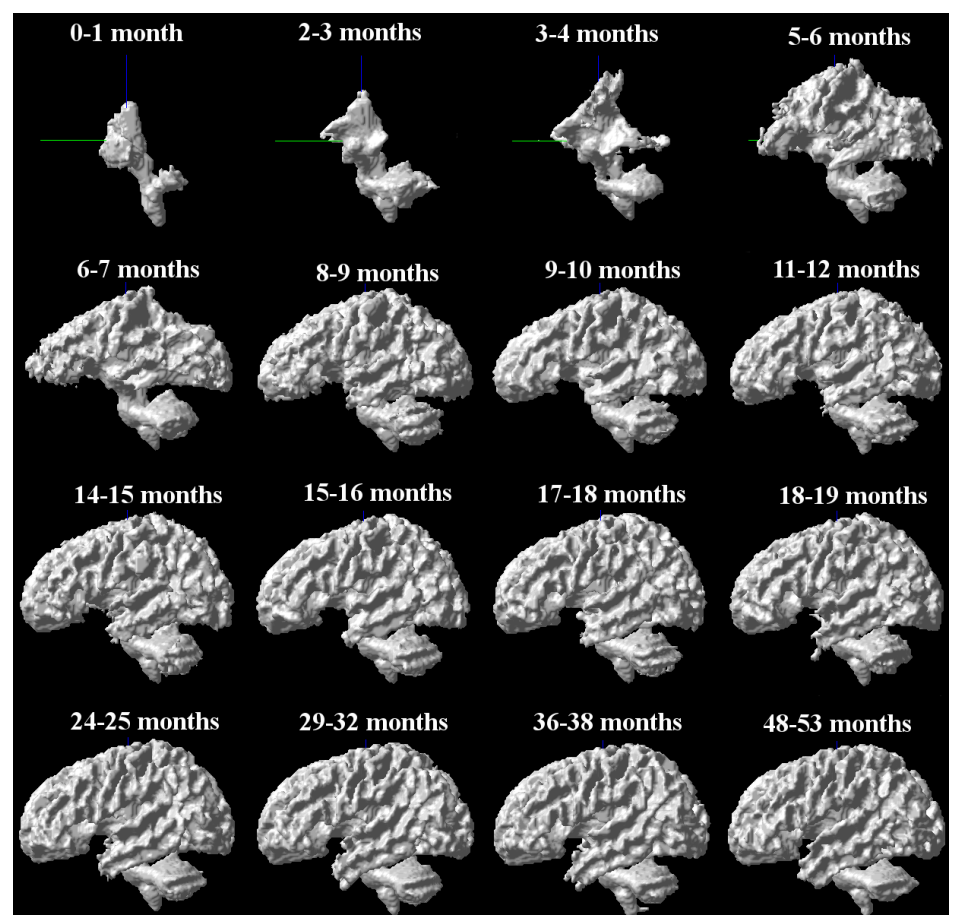

Fig. 5. Evolution of the myelinated WM from birth to 4 years 5 months

is always smaller after the cleaning. This shown how the cleaning step removes misclassified voxels and make the training set more homogeneous.

Some examples of brain classification are shown in Fig. 3 on one week to one year old infants. Usually, the classification works well on 6 or 8 months old brains and older, but before this age, the intensities of unmyelinated WM and cortical 
GM are very similar and the classification of these two structures is difficult (see for example the occipital classification on Fig. 3 (A) and (B)).

However, the classification of the myelinated WM, helped by the relaxometry data, gives consistent results. Voxel counting is used to estimate the volume of the myelinated WM volume in the standard space. Figure 4 shows the evolution of the myelinated WM volume for each subject from birth to 4 years 5 months.

For each month, the myelinated WM volume were averaged across subjects and surface models were obtained by triangulation of the averaged myelinated WM volumes with the marching cubes algorithm (Fig [5). The pattern described by Barkovich, commencing in the brain stem and progressing to the cerebellum and the cerebrum, proceeding from central to peripheral, from inferior to superior and from posterior to anterior, can be seen in the renderings.

\section{Discussion and Conclusion}

Our results confirm the topographical sequence of the development of myelination described in the literature [11415]: myelination proceeds from the central toward the superficial, and from the occipital and parietal lobes to the frontal and temporal lobes. A fast growth of the myelinated WM volume is observed between birth and 9 months of age. There is an important variation in myelinated volume between infants at the steepest part of the curve, from 5 to 7 months.

The automated classification has difficulties to separate WM and GM under 6 or 8 months of age, due to variable GM/WM tissue contrast. That is why, to our knowledge, there are no studies trying to classify the tissues after the neonatal period and before one year of age. However, the objective of our study was to focus on the myelinated WM classification and for this class, the classification results were consistent.

Differentiation between myelinated and unmyelinated WM was possible with the relaxometry data. Relaxometry is a quantitative imaging technique used to compute T2 relaxation times for each voxel in the brain. We know that the myelination is a continuous process; it depends on the number and the thickness of myelin sheats. It is therefore difficult to make a binary choice: myelinated or unmyelinated. In our case, the myelinated and unmyelinated WM differentiation depends on the choice of the threshold. If the value of the threshold is lower, more WM will appear as myelinated WM at a specific age and vice versa. We may therefore have small errors in the absolute timing of myelination. In this case, our classification gives an idea of the general development of the myelination but the age of the myelin appearance will be time-shifted depending on the threshold.

The accuracy of the detection should be computed by comparing the myelinated WM to a ground truth. As absolute truth is not possible with in vivo data, a manual classification is usually used for validation. However, in our case, a manual validation would be very subjective to the rater delineation of myelinated and unmyelinated structures.

In conclusion, this study represents the first 3D, longitudinal study to characterize myelination in a young normal pediatric population. The myelinated WM 
classification procedure can be used to study the normal brain development. This type of analysis opens the way to the development of new tools that could be used to detect demyelinating diseases in children.

\section{References}

1. Barkovich, A., Kjos, B., et al.: Normal maturation of the neonatal and infant brain: MR imaging at $1.5 \mathrm{~T}$. Radiology 166, 173-180 (1988)

2. Ono, J., Kodaka, R., et al.: Evaluation of myelination by means of the T2 value on magnetic resonance imaging. Brain Dev. 15, 433-438 (1993)

3. Nishida, M., Makris, N., et al.: Detailed semiautomated MRI based morphometry of the neonatal brain. Neuroimage 32, 1041-1049 (2006)

4. Gilmore, J., Lin, W., et al.: Regional GM growth, sexual dimorphism, and cerebral asymmetry in the neonatal brain. J. Neurosc. 27, 1255-1260 (2007)

5. Murgasova, M., Dyet, L., et al.: Segmentation of brain MRI in young children. Acad. Radiol. 14, 1350-1366 (2007)

6. Evans, A.: The NIH MRI study of normal brain development. NeuroImage 30, 184-202 (2006)

7. Sled, J., Zijdenbos, A., Evans, A.: A nonparametric method for automatic correction of intensity nonuniformity in MRI data. TMI 17, 87-97 (1998)

8. Coupe, P., Yger, P., et al.: An optimized blockwise non local means denoising filter for 3d magnetic resonance images. IEEE TMI (accepted, 2007)

9. Collins, D., Neelin, P., et al.: Automatic 3D intersubject registration of MR volumetric data in standardized Talairach space. In: JCAT, vol. 18, pp. 192-205 (1994)

10. Evans, A., Collins, D., et al.: 3D statistical neuroanatomical models from 305 MRI volumes. In: IEEE NSS/MIC, San Francisco, USA, pp. 1813-1817 (1993)

11. Cocosco, C., Zijdenbos, A., Evans, A.: A fully automatic and robust brain MRI tissue classification method. MIA 7, 513-527 (2003)

12. Ding, X., Kucinski, T., et al.: Normal brain maturation characterized with agerelated T2 relaxation times: an attempt to develop a quantitative imaging measure for clinical use. Invest Radiol. 39, 740-746 (2004)

13. Leppert, I., Almli, R., et al.: Pediatric age-related T2 relaxometry in normal brain development. In: OHBM, Florence, Italy (2006)

14. Van Der Knaap, M., Valk, J.: MR imaging of the various stages of normal myelination during the first year of life. Neurorad. 31, 459-470 (1990)

15. Parazzini, C., Baldoli, C., et al.: Terminal zones of myelination: MR evaluation of children aged 20-40 months. AJNR 23, 1669-1673 (2002) 